\title{
Ion Detachment in the Helicon Double-Layer Thruster Exhaust Beam
}

\author{
Fernando N. Gesto, ${ }^{*}$ Boyd D. Blackwell, ${ }^{\dagger}$ Christine Charles, ${ }^{\ddagger}$ and Rod W. Boswell ${ }^{\S}$ \\ Australian National University, Acton, Australian Capital Territory 0200, Australia
}

\begin{abstract}
This study is devoted to simulating the orbits of the ions in the supersonic beam observed experimentally in the laboratory development of the helicon double-layer thruster. Given the cylindrical symmetry of the problem, the particle orbits are generated in cylindrical coordinates $(r, \phi, z)$, thereby enabling the analysis of magnetic detachment (in which the particles free themselves from the magnetic field of the source) to be confined to the analysis of the curvature of trajectories on the $(r, z)$ plane. Because the trajectory of a magnetized particle in space is helical, detachment can be established if the curvature of $r(z)$ on the $(r, z)$ plane asymptotically approaches zero. The simulation shows that the detachment surface is a paraboloid opening in the direction of the expanding magnetic field, with its base along the radial $(r)$ axis oscillating around the axial value $z \approx 0.38 \mathrm{~m}$. The radius of the ion-beam detachment surface is in agreement with laboratory measurements of the beam density profile in the detachment region.
\end{abstract}

\begin{tabular}{ll} 
& \multicolumn{1}{c}{ Nomenclature } \\
$\boldsymbol{B}$ & $=$ magnetic field \\
$B_{r}$ & $=$ radial magnetic field \\
$B_{z}$ & $=$ axial magnetic field \\
$B_{\phi}$ & $=$ azimuthal magnetic field \\
$\hat{B}$ & $=$ unit vector along the magnetic field \\
$i$ & $=$ index of initial radial coordinate \\
$\boldsymbol{p}(s)$ & $=$ magnetic-field line trajectory \\
$r$ & $=$ radial coordinate \\
$r_{i}$ & $=$ initial radial coordinate of ion orbit \\
$s$ & $=$ distance along a magnetic-field line \\
$v_{\|}$ & $=$velocity parallel to the magnetic field \\
$v_{\perp}$ & $=$ velocity perpendicular to the magnetic field \\
$z$ & $=$ axial coordinate \\
$\phi$ & $=$ azimuthal coordinate
\end{tabular}

\section{Introduction}

$\mathbf{L}$ ABORATORY experiments have recently shown that currentfree electric double layers can be spontaneously created in helicon plasmas expanding in a diverging magnetic field. ${ }^{1-3}$ It was suggested that the ion beam measured downstream of the double layer could be used as a source of thrust in a new type of magnetoplasma space thruster presently defined as the helicon double-layer thruster (HDLT). ${ }^{1}$ Another type of magnetoplasma thruster is the variable specific impulse magnetoplasma rocket (VASIMR). ${ }^{4-6}$

Detachment of the plasma in the beam exhaust from the magnetic field produced by a magnetoplasma rocket is an element of thrust-

Received 12 October 2004; revision received 18 May 2005; accepted for publication 18 May 2005. Copyright (C) 2005 by the American Institute of Aeronautics and Astronautics, Inc. All rights reserved. Copies of this paper may be made for personal or internal use, on condition that the copier pay the $\$ 10.00$ per-copy fee to the Copyright Clearance Center, Inc., 222 Rosewood Drive, Danvers, MA 01923; include the code 0748-4658/06 \$10.00 in correspondence with the CCC.

${ }^{*}$ Graduate Research Student, Plasma Research Laboratory, Research School of Physical Sciences and Engineering; currently at School of Aerospace and Mechanical Engineering, University College, Australian Defence Force Academy, Australian Capital Territory 2600; fernando.gesto@ adfa.edu.au.

${ }^{\dagger}$ Senior Fellow, Plasma Research Laboratory, Research School of Physical Sciences and Engineering, H-1 NF National Plasma Fusion Facility.

${ }^{\ddagger}$ Senior Research Fellow, Plasma Research Laboratory, Research School of Physical Sciences and Engineering, Space Plasma and Plasma Processing Group.

${ }^{\S}$ Professor, Plasma Research Laboratory, Research School of Physical Sciences and Engineering, Space Plasma and Plasma Processing Group. vector analysis that involves experimental, analytic, and computational studies. The thrust in the HDLT prototype is generated by an ion beam that exits the nozzle of the thruster at supersonic velocity. ${ }^{7}$ The ion beam itself is generated by the formation, at the neck of the nozzle, of a current-free double-layer potential, ${ }^{1}$ which accelerates the ions generated in the helicon argon plasma.

In the laboratory (see Sec. II) the HDLT is attached to a diffusion chamber that is pumped down to simulate space. The accelerating potential difference is situated $25 \mathrm{~cm}$ along the nozzle $(z)$ axis, and with the present experimental setup this thrust mechanism is only observed $^{1}$ in a gas pressure range of 0.013 to $0.267 \mathrm{~Pa}$.

The solenoidal magnetic configuration encasing the helicon plasma source in the HDLT creates a "magnetic" nozzle, whose magnetic geometry in part determines the dynamics of the exhaust flow by influencing the orbits of the charged argon ions in the beam. The expanding magnetic field created by the solenoids influences the shape of the ion beam as it expands into space, giving the beam divergence and thereby affecting the net thrust generated by the beam. In the extreme case, where the magnetic-field intensity is high enough, the charged particles exiting the nozzle will be attached to the closed magnetic-field lines and no net thrust will be provided. At the other extreme, the beam velocity field will be determined mainly by the parallel acceleration across the double layer and will have almost no divergence, so thrust will be generated at maximum efficiency.

The aim of this study is to analyze the trajectories of the HDLT exhaust ion particles in the expanding magnetic field created by the solenoids surrounding the plasma source. In particular, the simulation is used to analyze whether the accelerated ions in the laboratory prototype "detach" from the magnetic field to provide thrust and what influence the magnetic-field strength has on the detachment process. The simulation is particularly important for the understanding of the behavior of the beam exhaust at distances from the nozzle that currently exceed the axial-length scale of the laboratory experiment.

We introduce a new geometric approach to analyzing the magnetic detachment of the ion beam. Traces of the magnetic field and particle trajectories, for different initial radial values $r_{i}$ across the beam's radial cross section at the beam origin, are generated in cylindrical coordinates and traced onto the $(r, z)$ plane. From the particle trajectory traces, the curvature of the orbits $r_{i}(z)$ is calculated. We then analyze 1) whether the ion motion detaches from the influence of the magnetic field and 2) at what point in its trajectory it detaches.

A charged particle is unmagnetized, or detached, if its trajectory has zero curvature. Given that the curvature of motion of a charged particle in a magnetic field may asymptotically approach 